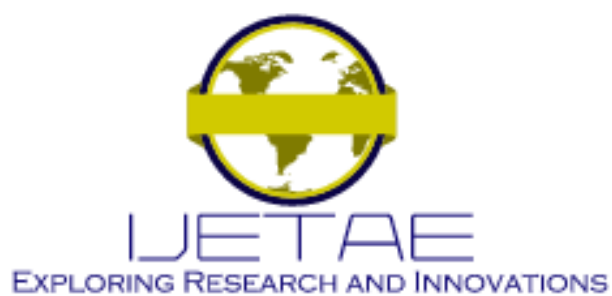

International Journal of Emerging Technology and Advanced Engineering

Website: www.ijetae.com (E-ISSN 2250-2459, Scopus Indexed, ISO 9001:2008 Certified Journal, Volume 11, Issue 06, June 2021)

\title{
An Efficient Parallel Architecture for Sub Band Processor in Broad Band MVDR Beamformer for Medical Ultrasound Applications
}

\author{
Sreejeesh $\mathrm{SG}^{1}$, Sakthivel $\mathrm{R}^{2}$ \\ ${ }^{I}$ National Institute of Electronics and Information Technology, NIT Campus Post, Calicut 673601. \\ ${ }^{2}$ Vellore Institute of Technology, Katpadi, 632014. (Corresponding Author)
}

\begin{abstract}
Minimum Variance Distortion less Response (MVDR) algorithm is the key adaptive beamforming algorithm in high resolution Medical Ultrasound Imaging systems. MVDR Beamformer is a frequency domain beamformer, and each frequency sub band need to be processed to generate covariance matrix and the same is computationally costly. Though many VLSI architectures have been proposed for MVDR Beamformer, currently no Complex Correlation matrix generation architecture for frequency sub band has been devised which supports real time implementation of broad band MVDR Beamformer for Medical Ultrasound Imaging. In this paper we show real time MVDR sub array, sub band processor realization for an array with number of elements $\mathrm{N}=32$ and sub array length $\mathrm{L}=8$. To satisfy the narrow band condition of the array we employed $1 \mathrm{~K}$ point FFT, with $12.5 \%$ overlap. The entire design of MVDR Sub band processor is tested on the Field Programmable Gate Array (FPGA) platform. The implementation results show that the through put of the developed architecture is $600 \mathrm{~K}$ Complex Correlation matrix generation per second which is equivalent to $65.57 \mathrm{fps}$ to image a $20 \mathrm{~cm}$ depth target at a Pulse Repetition Frequency (PRF) of $4 \mathrm{KHz}, 60^{\circ}$ Field of View and $1^{\circ}$ lateral resolution and makes the MVDR a viable solution for practical systems.
\end{abstract}

Keywords - MVDR Beamformer, Subband Processor, Sub Array Beamforming, Coorelation Matrix, VLSI Architecture, Medical Ultrasound.

\section{INTRODUCTION}

Broadband MVDR Beamformer consists of various computational blocks such as FFT processor, FFT sub band processor to generate correlation matrix, correlation matrix inversion, adaptive weight computation ,output computation and inverse FFT processor[1][2].
Among the computational processing blocks FFT Sub band processor and Correlation matrix inversion [3] are computationally intensive. Implementing those processing blocks in DSP processor is hard to meet, the timing constraints imposed by real time ultrasound imaging systems.

Recent works [4][5] demonstrates GPU as embedded implementation platform for MVDR beamformer implementation, but ultrasound imaging systems lacks in adapting GPUs due to its $\mathrm{I} / \mathrm{O}$ data issues. Therefore to meet the real time requirements of medical ultrasound imaging systems, VLSI implementation of these processing blocks are imperative. In the past decades starting from Frost [3], several researchers have studied the MV beamformer for ultrasound imaging. Previous studies have reported that MV beamformers show significantly improved resolution and interference suppression capability over the existing Delay and Sum (DAS) beamformer [6][7][8]. However, the improvement comes at the expense of increased computational cost from the calculation of the spatial covariance matrix and its inverse, which limits the use of the MV beamformer in practice. Also the ultrasound echoes exhibits broad band characteristics and MVDR performance degrades in broad band scenario. Hence broad band MVDR implementation is recommended.

The paper is structured as follows:

Section II summarizes the existing implementations and an overview of MVDR Beamformer FFT Sub Band Processor. Section III describes the VLSI Architecture Design for the Sub Band Processor, Section IV discuss about the results and its implementations, section $\mathrm{V}$ gives the conclusion of the article and section VI discusses the limitations of the implementations and future scopes. 


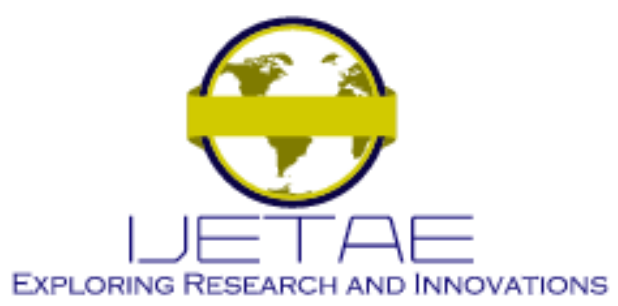

International Journal of Emerging Technology and Advanced Engineering

Website: www.ijetae.com (E-ISSN 2250-2459, Scopus Indexed, ISO 9001:2008 Certified Journal, Volume 11, Issue 06, June 2021)

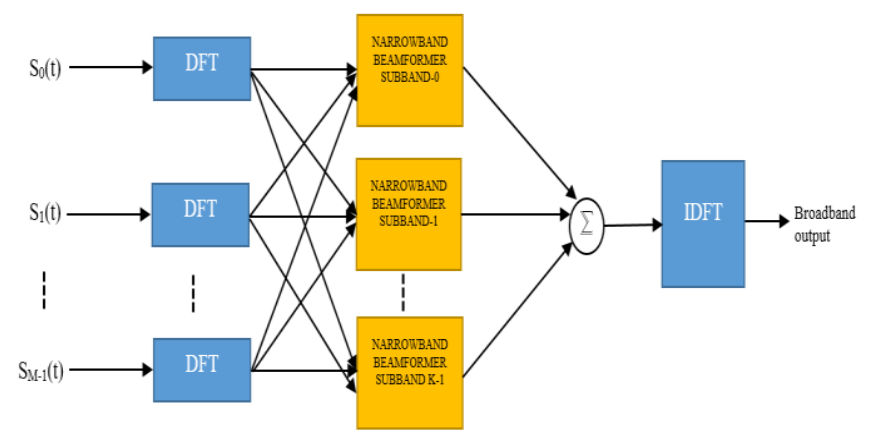

Fig. 1 Block diagram of Broad band MVDR beam former.

\section{BACKGROUND}

\section{A. Literature Survey - Broad band MVDR Beamformer}

We will have a brief literature survey about various beamformers available in field. Fig. 1 depicts the functional block diagram of Board band MVDR beamformer. Using Discrete Fourier Transform (DFT) the samples from ' $M$ ' sensors are transformed into frequency domain. Each DFT output points (frequency bins) are narrow sub bands, sub band beamforming is performed for each sub band from ' $\mathrm{M}$ ' channels. The time domain data is retrieved from each sub band by using inverse DFT, all beamformed outputs are added and given to inverse DFT block. In Medical Ultrasound Imaging system, beam formed output in time domain is essential to perform further processing and to generate the image. To employ this computationally intensive algorithm for real time Medical Ultrasound Imaging, a dedicated hardware for various processing blocks involving parallelism may be a good alternative. In FFT Sub band beamforming ' $M$ ' channel outputs can process in parallel and hence parallelism can be easily adapted.

MVDR Beamformer works based on the principle that, minimizes the power in particular look direction under the constraint, the desired signal passes without any distortion (1). This will result in suppressing the side lobes which will improve signal to interference ratio. Fig. 2 depicts the detailed broad band MVDR Beamformer implementation block diagram for Kth sub band. In Broad band MVDR Beamformer we used a N-point FFT to spilt the bands of frequencies into narrow sub bands. The equation (2) computes the complex correlation matrix $\mathrm{R}$ which corresponds to each sub band. The respective weights which are adaptive in nature are calculated by using equation (3).
The final output is computed with the help of equation (4). The output of each bin is calculated and stored, we next process N-bins and all the values of output are given to the IFFT block. This block performs its operation and provides the system with time domain data. A detailed overview of the MVDR Beamformer is briefly explained in the article [9].

MVDR Beamformers are basically used for medical ultrasound imaging applications. MVDR Beamformer [10] needs to update the beamformer weights continuous. The need of performing this is to minimize the power of beamformer output, at the same time it should enhance the beam response from the focal point. Because medical ultrasound data has broad band characteristics, the array's narrow band criterion must be met. The FFT slice size should be designed in such a manner that it meets the array's narrow band requirements.

$$
\begin{gathered}
\text { Min } \mathrm{W}^{\mathrm{H}} \mathrm{RW} \\
\text { Condition is } \mathrm{W}^{\mathrm{H}} \mathrm{S}(\theta)=1 \\
\mathrm{R}=\mathrm{E}\left[\mathrm{XX}^{\mathrm{K}}\right] \\
\frac{R^{-1} S(\theta)}{S(\theta)^{H} R^{-1} S(\theta)} \\
\mathrm{Y}_{\mathrm{K}}(\theta)=\mathrm{W}_{\mathrm{K}}(\theta)^{\mathrm{H}} \mathrm{X}_{\mathrm{K}}
\end{gathered}
$$

\section{B. Sub Array MVDR Beamforming}

The covariance matrix is unknown in real-time applications and therefore should be constructed from data. The computational burden of constructing the spatial covariance matrix and its inverse hinders the employment of the MVDR Beamformer in real-time applications like as medical ultrasound applications. When considering realtime implementation, the construction and inversion of covariance matrices become a mammoth effort. In sub array MVDR, the transducer array is divided into overlapping sub arrays to decrease the size of the spatial covariance matrix, and the covariance matrices for each sub array are averaged throughout the array, resulting in a covariance matrix and its inverse that is practically implementable. Fig. 3 depicts sub array averaging technique. The transducer array is converted into ' $\mathrm{P}$ ' $(\mathrm{P}=\mathrm{M}-\mathrm{L}+1)$ sub arrays, these arrays overlap each other our next step is to compute the covariance and that is averaged across the array. It is also known as spatial smoothing and the covariance matrix is computed by (5). 


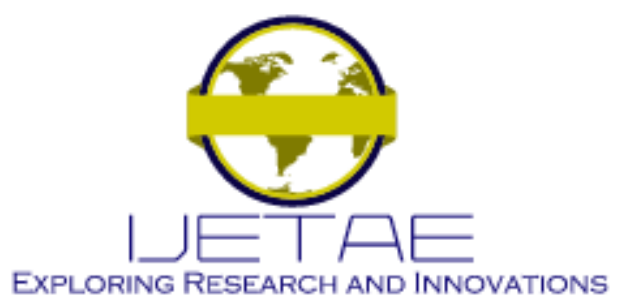

International Journal of Emerging Technology and Advanced Engineering

Website: www.ijetae.com (E-ISSN 2250-2459, Scopus Indexed, ISO 9001:2008 Certified Journal, Volume 11, Issue 06, June 2021)

The covariance matrix $R^{\wedge}(\omega)$ is averaged across each frequency sub band and the size of covariance matrix is reduced to $\mathrm{L} \times \mathrm{L}$ instead of $\mathrm{M} \times \mathrm{M}$ and the spatially smoothed covariance matrix estimate will always become non-singular, if the size of the sub array satisfies, $\mathrm{L} \leq \frac{M}{2}$.

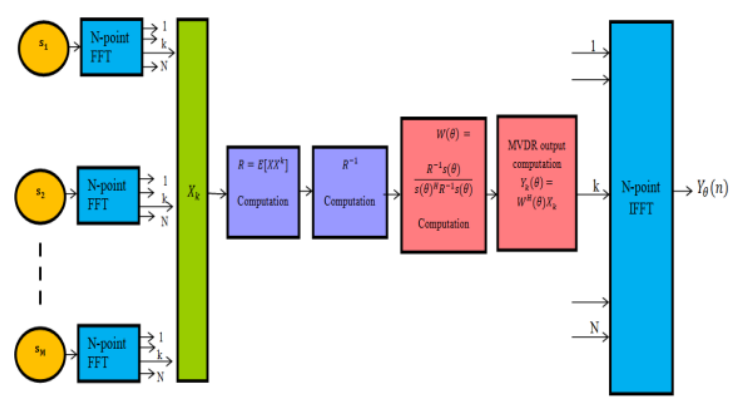

Fig. 2 Broad Band MVDR Beamformer Implementation.

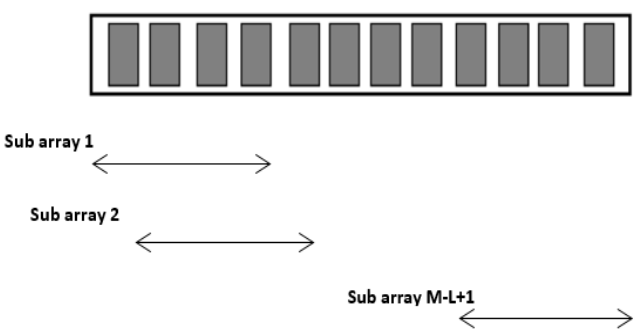

Fig. 3 Sub Arrary Avergaing .

$$
\begin{array}{r}
R^{\wedge}(\omega)=\frac{1}{M-L-1} \sum_{l=0}^{M-L} E\left[x_{l}(\omega) x_{l}(\omega)^{H}\right] \\
\text { Where } x_{l}[\omega]=\left[\begin{array}{c}
x_{l}[\omega] \\
x_{l+1}[\omega] \\
\vdots \\
x_{l+L-1}[\omega]
\end{array}\right]
\end{array}
$$

For $\mathrm{l}=0,1 \ldots \mathrm{M}-\mathrm{L}$

Where $\mathrm{M}$ is the number of sensors and $\mathrm{L}$ is the length of the sub array.

\section{Overlapped FFT}

To improve the beamformer response, coherency, overlapped FFT need to be performed while employing frequency domain beamforming. The narrow band condition of a transducer array is illustrated in (6).

$$
\left(\frac{f_{s}}{N_{f f t}}\right) \tau_{\max }<<<1
$$

Where $\tau_{\max }$ is the maximum travel time across the array, i.e. $\mathrm{L} / \mathrm{c}$ where ' $\mathrm{L}$ ' is the length of the array and ' $c$ ' is the speed of ultrasound sound wave. Hence the number of sample overlap should be greater than or equal to $\tau_{\max } * f_{s}$. The $N_{f t t}$ also will be fixed based on (6). Suppose we are performing $\mathrm{N}$ point FFT with $\mathrm{M}$ sample overlap, the time required to digitize N-M samples defines the shortest resolvable time event as real time implementation is concerned.

\section{Computational Complexity}

The computational complexity to estimate correlation matrix R, in sub array broad band MVDR beamformer is $(\mathrm{N} / 2)(\mathrm{M}-\mathrm{L})(\mathrm{L} X \mathrm{~L})+\mathrm{N} / 2$ complex multiplications and $(\mathrm{N} / 2)(\mathrm{M}-\mathrm{L}-1)$ complex additions. Where $\mathrm{M}$ is the number of channels, $\mathrm{L}$ is the sub array size and $\mathrm{N}$ is the number of FFT points. The sub array beamforming algorithm discussed, need to compute N/2 complex correlation matrixes and per sub band M-L matrixes need to be computed. To compute R per each sub band L X L complex multiplications and M-L-1 complex additions need to be performed. Hence as per sub array algorithm discussed $(\mathrm{N} / 2)(\mathrm{M}-\mathrm{L})(\mathrm{L} X \mathrm{~L})+\mathrm{N} / 2$ complex multiplications and (N/2)(M-L-1) complex additions need to be performed to estimate $\mathrm{R}$. Computing $\mathrm{R}$ is hence the most complex operation in sub array MVDR beamformer. Next we discuss modeling sub array MVDR in MATLAB and mapping of this arithmetic complexity in to a parallel VLSI architecture.

\section{E. Sub Array MVDR Model}

To test the functionality of the developed VLSI architecture, a golden reference model is developed in MATLAB using Field II tool box [11].Table-1 illustrates the specifications followed. 


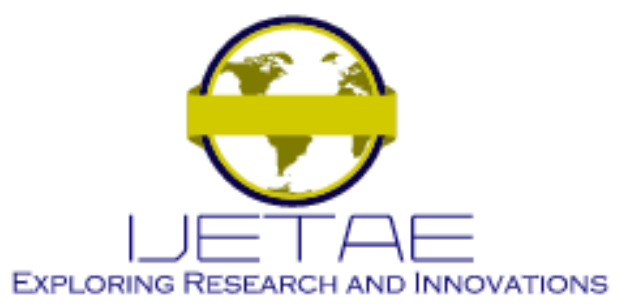

International Journal of Emerging Technology and Advanced Engineering

Website: www.ijetae.com (E-ISSN 2250-2459, Scopus Indexed, ISO 9001:2008 Certified Journal, Volume 11, Issue 06, June 2021)

Table-1

Specification of Sub Band Processor in MVDR Beamformer.

\begin{tabular}{|c|c|}
\hline Parameters & Values \\
\hline No. of elements in transducer, $\mathrm{N}$ & 32 \\
\hline Sampling frequency, Fs & $20 \mathrm{MHz}$ \\
\hline Velocity, C & $1540 \mathrm{~m} / \mathrm{s}$ \\
\hline Central frequency, f0 & $4 \mathrm{MHz}$ \\
\hline Sub array length, L & 8 \\
\hline Wave length, $\lambda$ & $385 \mu \mathrm{m}$ \\
\hline Pitch & $192.5 \mu \mathrm{m}$ \\
\hline Kerf & $9.625 \mu \mathrm{m}$ \\
\hline Width $=$ Pitch - Kerf & $182.85 \mu \mathrm{m}$ \\
\hline Element Height & $10 \mathrm{~mm}$ \\
\hline Sector angle & $30^{\circ}$ \\
\hline Length of the array & $0.5967 \mathrm{~cm}$ \\
\hline$\tau_{\max }$ & 3.87 \\
\hline Bandwidth & $<0.25 \mathrm{MHz}$ \\
\hline No of point FFT & 1024 \\
\hline No of sample overlap & 128 \\
\hline
\end{tabular}

We considered the number of elements ' $\mathrm{N}$ ' as 32 and sub array length ' $\mathrm{L}$ ' as 8 . A $4 \mathrm{MHz}$ linear array with $\lambda / 2$ spacing was modeled in simulation.
The length of the array is (N-1) x Pitch which is calculated as $0.59 \mathrm{~cm}$ and the travel time across the array is calculated as $3.87 \mu \mathrm{s}$. For practical consideration, the narrow band condition of the array is considered as (7). As sampling frequency chosen was $20 \mathrm{MHz}$ and $\tau_{\max }$ is $3.87 \mu \mathrm{s}$, to satisfy (7) the number of point FFT was chosen as 1024 , and by considering the travel time across the array, the number of sample overlap was taken as 128 .

$$
\left(\frac{f_{s}}{N_{f f t}}\right) \tau_{\max }<<<0.1
$$

An 8 cyst phantom positioned at $20 \mathrm{~mm}, 21 \mathrm{~mm}, 22 \mathrm{~mm}$, $50 \mathrm{~mm}, 60 \mathrm{~mm}, 70 \mathrm{~mm}, 80 \mathrm{~mm}$ and $90 \mathrm{~mm}$ is modeled using Filed II tool box, focused at $60 \mathrm{~mm}$ depth and ultrasound echo was generated by energizing the modeled transducer.

Each channel output was buffered for FFT segment size $(1 \mathrm{~K})$, overlapped FFT is implemented with an overlap of 128 sample, and hence there was 896 new samples. The same is depicted in article [12] with a superior implementation technique. Time to process $1 \mathrm{~K}$ samples were considered as the real time requirement of the MVDR Beamformer, and here it is $51.3 \mu$ sce. The real time implementation techniques are very precisely explained in [13] Table-2 illustrates the sub array MVDR Beamformer algorithm realized in MALAB. VLSI Architecture of QR Array Processor and its implementation [14] was studied and used as a reference in arriving at the new architecture.

For achieving the minimum frame rate and better resolution a minimum-variance beamformer implementation techquie was introduced in articles [15][16][17]. Here the side lobe removal method is effectually used. To improve the resolution adaptive methods have evolved and beam width is confined to narrow for all these methods to improve the various properties of image in ultrasound imaging. The minimumvariance beamformer and MVDR beamformer implementation [18] is suggested by many articles upon the classic methods like delay-and-sum, these improved methods produced better frame rates, improved aperture without deteriorating the image quality. 


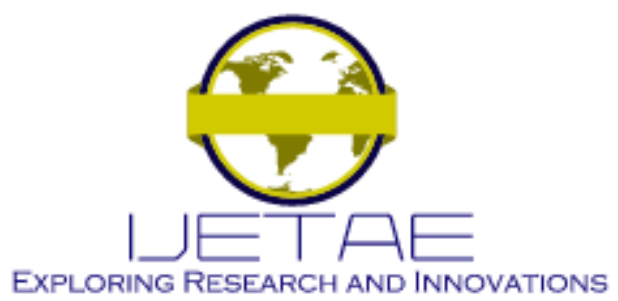

International Journal of Emerging Technology and Advanced Engineering

Website: www.ijetae.com (E-ISSN 2250-2459, Scopus Indexed, ISO 9001:2008 Certified Journal, Volume 11, Issue 06, June 2021)

Table-2

Sub array MVDR Beamformer Algorithm

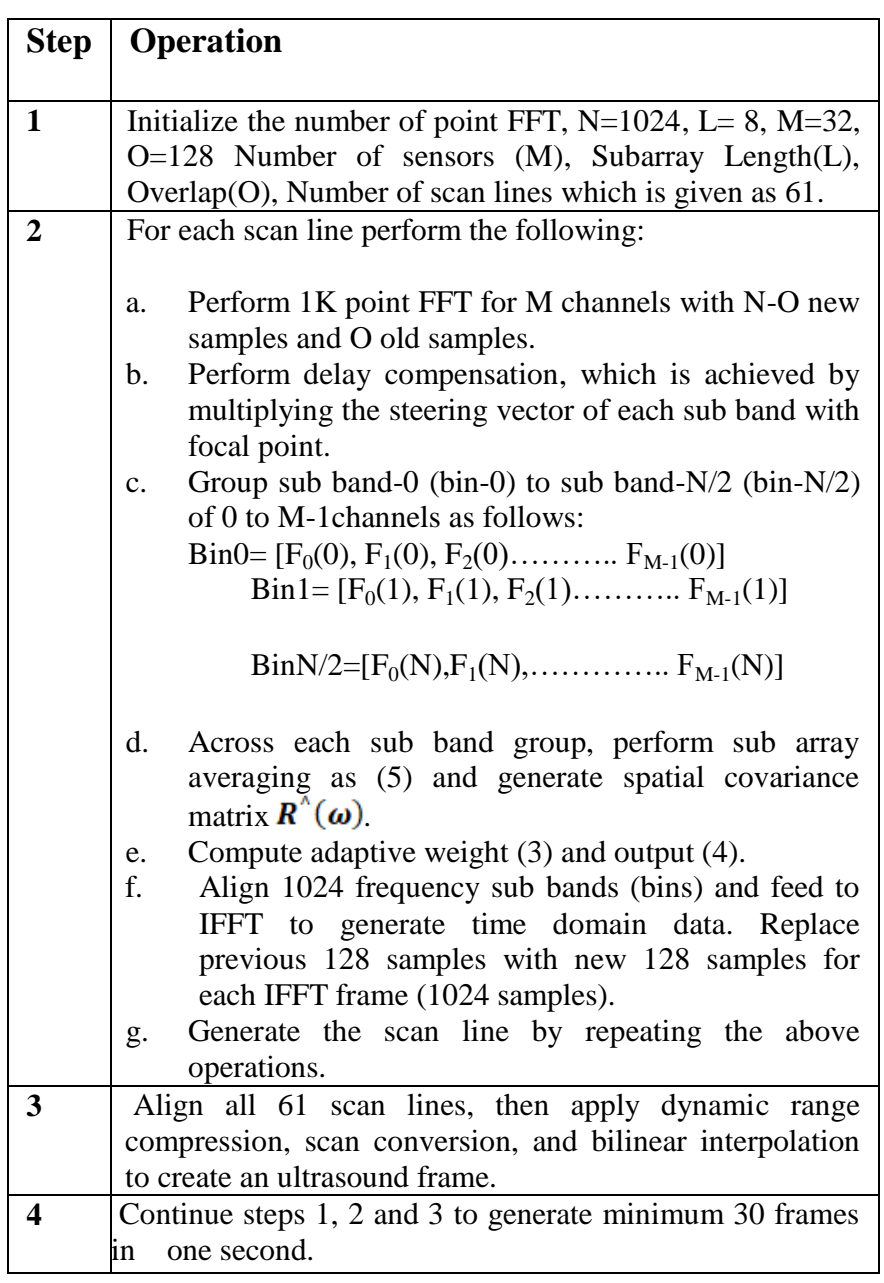

\section{VLSI ARCHITECTURE DESIGN}

In this section we will derive a parallel VLSI architecture of MVDR Sub array Beamformer (Fig.4). The architecture employs two stage pipeline; FFT processor and bin processor for computing correlation matrix. The digital samples from Analog Frontend (AFE) are stored in a $1 \mathrm{~K}$ size circular buffer for real time streaming. The channel memory controller reads 896 new samples and 128 old samples and streams to $1 \mathrm{~K}$ point FFT. 32 FFTs are performed in parallel to process 32 channels simultaneously.
The FFT controller feds the FFT out puts to delay compensation module, it also generates the necessary control and address signals to steering vector memory. The delay compensation module multiplies each channel FFT bin with corresponding steering vector values to compensate the delay from a focal point for each channel. Further it writes the delay compensated output to a ping pong channel memory. It has real and imaginary memory banks. The R-sequencer reads a particular bin value for each channel and fed to R-computation processor. The Rcomputation processor computes 24 correlation matrix in parallel as required by the real time sub array MVDR algorithm [19]. The R-averager generates an average correlation matrix out of 24 matrixes per bin. Finally the Rmemory controller writes the averaged correlation matrix of each channel bin in correlation matrix (R-memory), which is also a ping pong complex memory. The master controller synchronizes the sequence of operations among sub control units in the proposed sub band processor architecture. The proposed architecture has memory units, control units and processing units.

\section{A. Memory Unit}

The memory unit has various memory modules which are input channel memory, steering vector memory, FFT memory and correlation matrix memory. The input channel memory units are circular buffers, FFT and Correlation matrix memory units are ping pong buffers. Thirty two 1024 x 16 circular buffers are employed at the input for real time streaming of analog frontend data. Circular buffers are employed at the input side for the ease of real time overlapped FFT implementation. To avoid any data miss during real time processing ping pong buffers are adapted for FFT memory which stores the frequency domain data and correlation matrix memory which stores the generated correlation matrix. Sixty one steering vectors of $32 \mathrm{X} 1$ column vector of 16 bit wide is stored in steering vector memory. To improve memory access bandwidth steering vectors are arranged as depicted in Fig.5, hence in one clock one steering vector can be read. Table- 2 shows the memory requirement for the sub band processor. For input channel memory $2 \mathrm{~K}$ Bytes per channel i.e $64 \mathrm{~KB}$ for 32 channels are utilized, to store 61 steering vectors of $32 \times 1$, 3.7 KB of memory and to store FFT output, Correlation matrix $128 \mathrm{~KB}$ each is utilized. The memory foot print is optimized by selecting input channel memory as circular buffer and considering the symmetry property of FFT i.e only 512 bins are stored for processing. 


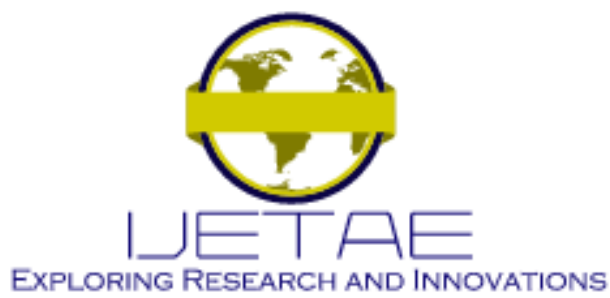

International Journal of Emerging Technology and Advanced Engineering

Website: www.ijetae.com (E-ISSN 2250-2459, Scopus Indexed, ISO 9001:2008 Certified Journal, Volume 11, Issue 06, June 2021)

Table-3

Memory Requirement of Sub Band Processor

\begin{tabular}{|c|c|}
\hline Memory Unit & Size in KB \\
\hline Input Channel Memory & 64 \\
\hline Steering Vector Memory & 3.7 \\
\hline FFT Output Memory & 128 \\
\hline Correlation Matrix Memory & 128 \\
\hline Total & 323.7 \\
\hline
\end{tabular}

B. Control Unit

The main controllers in sub band processor are channel memory controller, FFT Controller, R-sequencer, Rmemory controller and master controller. The controllers are implemented as Mealy FSMs, the channel memory controller has 4 states, INIT state, S0, S1 and S2. Moment start signal is asserted the controller moves from INIT state to S0 state. In S0 state the controller waits till Ready For Data (RFD) signal of FFT processor is high. Whenever RFD is high the FFT Processor asserts address out and the controller state moves from S0 to S1. In S1 sate, the circular buffer address will be generated by adding FFT processor output address bus to 896. Hence the FFT Processor [20] starts fetching data from 896 onwards, i.e. 128 old samples and 896 new samples for $12.5 \%$ overlap. 


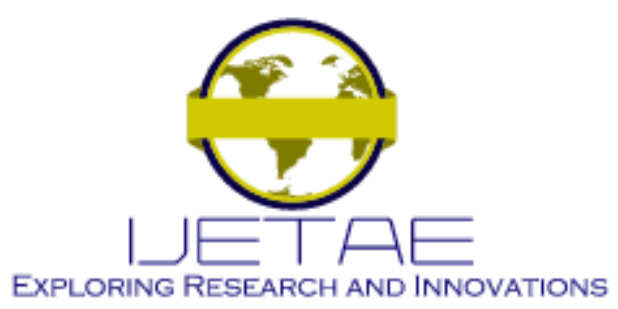

International Journal of Emerging Technology and Advanced Engineering

Website: www.ijetae.com (E-ISSN 2250-2459, Scopus Indexed, ISO 9001:2008 Certified Journal, Volume 11, Issue 06, June 2021)

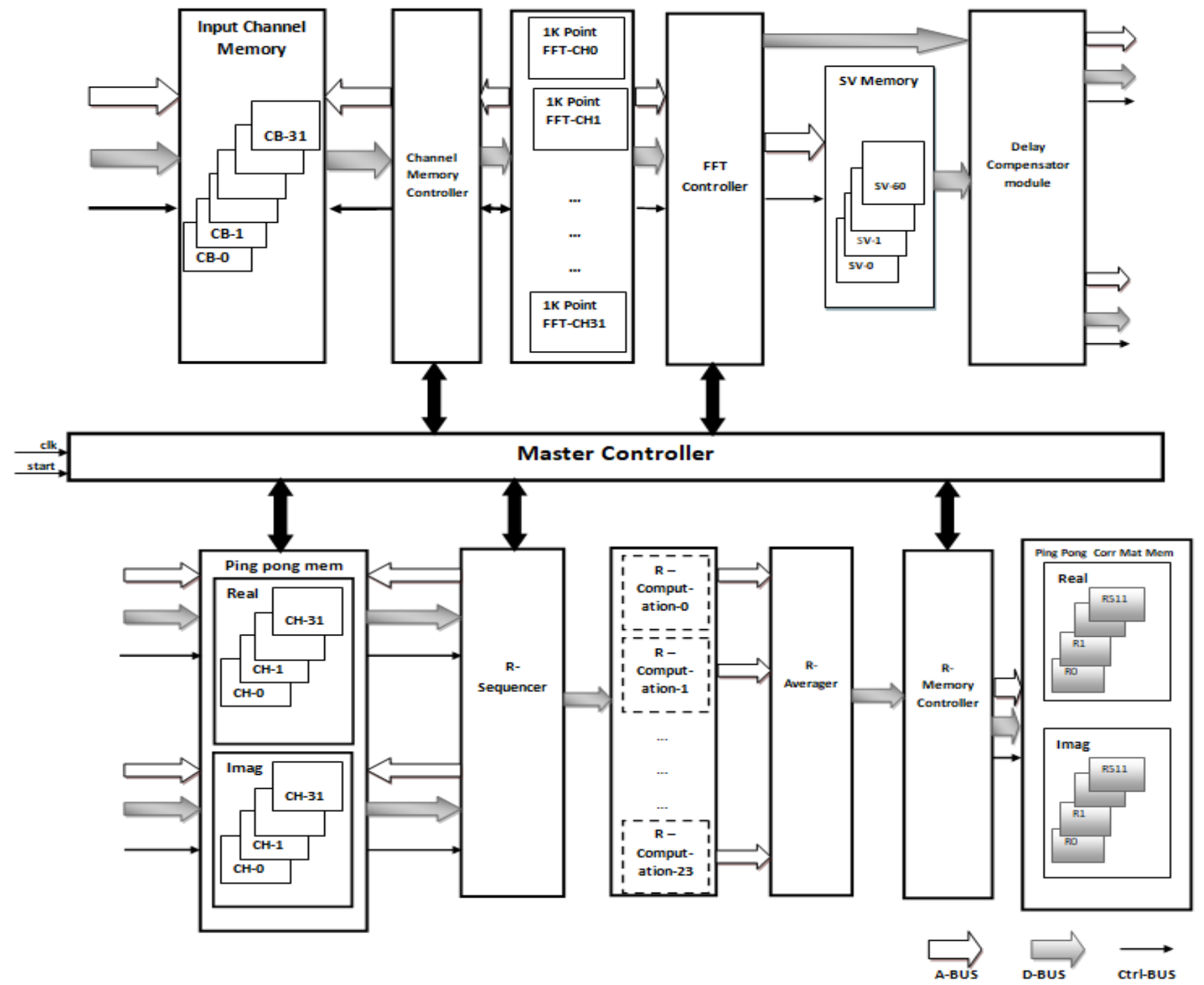

Fig.4 FFT Sub band Processor.

Moment one scan line processing ends the state moves to $\mathrm{S} 2$ and continues in $\mathrm{S} 2$ till freeze signal is activated. Freeze is the control signal of a typical ultrasound system to pause the scanning process.

The FFT Controller FSM works similar to channel controller FSM in which, moment the FFT output data valid signal is valid, the controller moves from INIT state to S0 state. In S0 state the FFT output is delayed by one clock cycle, the steering vector memory address is generated based on steering angle control from master controller and state moves to S1.
In S1 state the steering vector memory data, FFT output will be streamed to complex multiplier as well as the steering vector address will be incremented. Further in state S1 the FFT output data and steering vector data will be ready and streamed to delay compensator module. It continues in state $\mathrm{S} 1$ until, freeze signal asserts. Once freeze signal is asserted the state moves to INIT and waits for the next FFT output data valid signal to continue the process. 


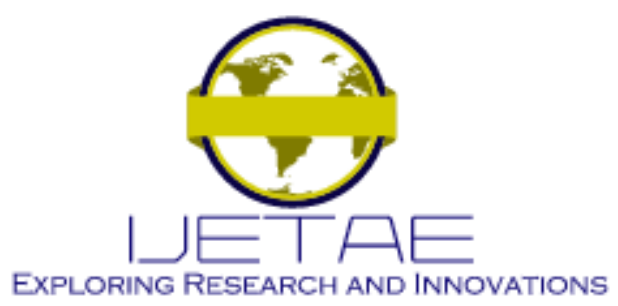

International Journal of Emerging Technology and Advanced Engineering

Website: www.ijetae.com (E-ISSN 2250-2459, Scopus Indexed, ISO 9001:2008 Certified Journal, Volume 11, Issue 06, June 2021)

The R-sequencer reads the frequency domain data i.e. real and imaginary FFT output memory and fed to RComputation block. Which computes sub array correlation matrix R. The R-sequencer is implemented in 4 states INIT, $\mathrm{S} 0, \mathrm{~S} 1$ and $\mathrm{S} 2$. Once the data is written to ping pong FFT memory, R-data ready signal will be asserted by the master controller and R-sequencer FSM moves to S0 state. In S0 state, the sequencer issues read signal to FFT memory and moves to S1 state. In S1 state bin-X (x varies from 0 to 511) data for all 32 channels will be ready, and the same is streamed to R-computation processor. It continues in $\mathrm{S} 1$ state till bin counter is 511 , which is provided by the master controller. When bin counter reaches to 511 , it moves to $\mathrm{S} 2$ state where $511^{\text {th }}$ bin data is send to R-Computation processor, further it moves to S0 state and continues till freeze signal is activated. Once freeze signal is activated the controller moves back to INIT state till next R-data is ready.

The R-memory controller is implemented in two states INIT and S0. Moment R averager output is valid it moves from INIT state to S0 state. S0 state has an in build cyclic FSM which operates at $160 \mathrm{MHz}$ and counts from zero to seven. It is implemented in such a way, due to the $\mathrm{R}$ memory requires 8 clock cycles to write the $\mathrm{R}$ averager output.

The master controller implemented has sample_cnt which counts for every sampling clock, scanline_cnt which increments for every Pulse Repetition Interval, bin_cnt which counts from 0 to 511 within one fftoverlap_cnt and frame_cnt which increments when scanline_cnt reaches 60, i.e. for every frame. The master controller sequences the data and control flow among FFT processor and bin processor. The master controller is implemented in six states; INIT, S0, S1, S2, S3 and S4. Moment start signal is activated, the controller moves from INIT state to S0 state, where sample_cnt increments. Moment sample_cnt reaches 5194, which corresponds up to $20 \mathrm{~cm}$ depth samples, the state moves to S1 and scanline_cnt increments. For every even sample_cnt the bin_cnt increments and it is in sync with FFT overlap_cnt state S3. In state S4 the frame_cnt increments, corresponds to 61 scan lines per frame. The controller generates the required control signals to various processing blocks in sub band processor till freeze signal is activated.

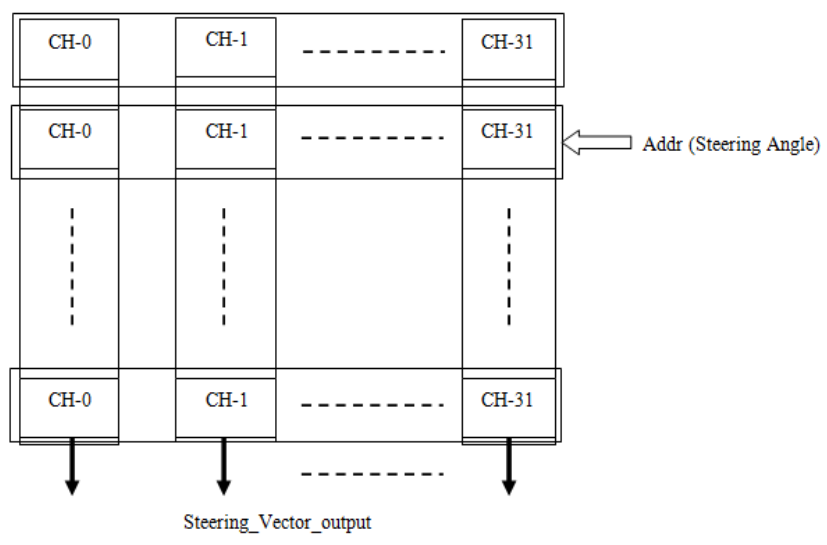

Figure.5-Steering Vector Memory Bank

\section{Data path Units}

The main data path units in sub band processor data paths are FFT, R-Computation processor and R-averager.

In this work we have employed Xilinx ${ }^{\mathrm{TM}}$ FFT IP Core [12]. The IP Core was configured as Radix-2, 1024 point, pipelined streaming mode, operates at $200 \mathrm{MHz}$. In order to compute 1024 point FFT it takes 2000 clock cycles i.e. approximately $16 \mu \mathrm{sec}$. As per the implemented specification 32 channels are processed in parallel, thirty two FFTs are used each having different controller. Finally the FFT output is stored in another DPRAM. Since FFT has real and imaginary values, each channel requires two different DPRAMs. By considering the symmetric property of FFT, only 512 samples are taken from 1024 samples that is, since after every $(\mathrm{N} / 2+1)$ values, FFT will repeat the output.

The R-computation processor computes the correlation matrix as in equation (5). Where $\mathrm{E}$ denotes expectation of $\{$.$\} and X$ is the column vector of FFT Output in each sub array. Assume the process is ergodic, where the ensemble average equals the time average, then $\mathrm{R}$ becomes as referred in Equation (8),

$\widehat{R}[n]=\frac{1}{M-L+1} \sum_{l=0}^{M-L} x_{l}[n] x_{l}^{H}[n]$

Where $x$ is a [8 $\mathrm{x} 1$ 1] complex column vector read from the real and imaginary memories of FFT output. $R$ is a $[8 \mathrm{x}$ 8] complex matrix for $M-L+1$ sub arrays and finally the $R$ for each bin is averaged. 


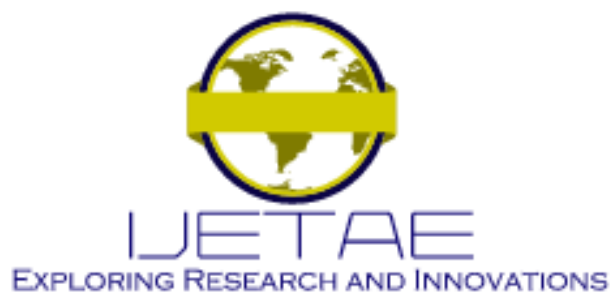

International Journal of Emerging Technology and Advanced Engineering

Website: www.ijetae.com (E-ISSN 2250-2459, Scopus Indexed, ISO 9001:2008 Certified Journal, Volume 11, Issue 06, June 2021)

This repeats for to FFT bins $0,1,2 \ldots 512$ and given for further processing. The R-Computation requires one complete column of a sub array correlation matrix to be generated in a single clock. Therefore 8 parallel complex multiplier blocks are realized, which is depicted in Fig. 6.Therefore in 8 clock cycles one $8 \times 8$ matrix will be computed. Since 24 matrices are computed in parallel, in 8 clock cycles correlation matrix per bin will be computed. The R-averager block, performs addition and spatial averaging of all 24 correlation matrixes. The spatially added R-matrix is averaged by $1 / \mathrm{M}-\mathrm{L}+1$, which is $1 / 25$ in our design. Since our design is for dedicated architecture, we represented 1/25 in Q15 fixed point format $(0 \times 51 F)$ and multiplied with spatially generated R-matrix.

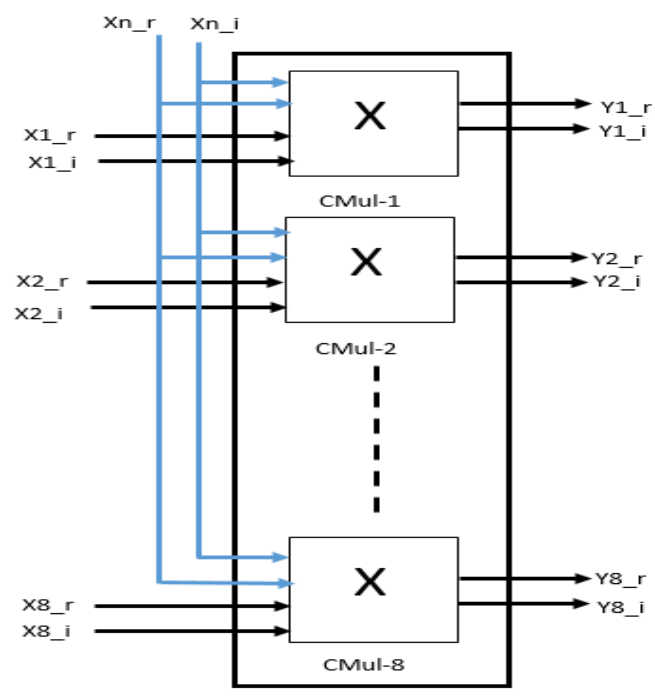

Fig. 6 R-Computation processor.

\section{RESUlts AND Discussions}

Our design has been tested against the Sub Array MVDR Model developed in MATLAB. The rf data generated for each channel using MATLAB Field II Tool box were converted into 1.15 fixed point format and loaded into circular buffers for processing. For accurate results, the processed data, i.e. correlation matrix need to be represented with higher number of bits, and here we represented with 1.63 fixed point format. The correlation matrix generated for each bin for each steering angle from FPGA simulation results and MATLAB counterpart were compared and the error was in -90 to -140 (Fig.7) dB range.

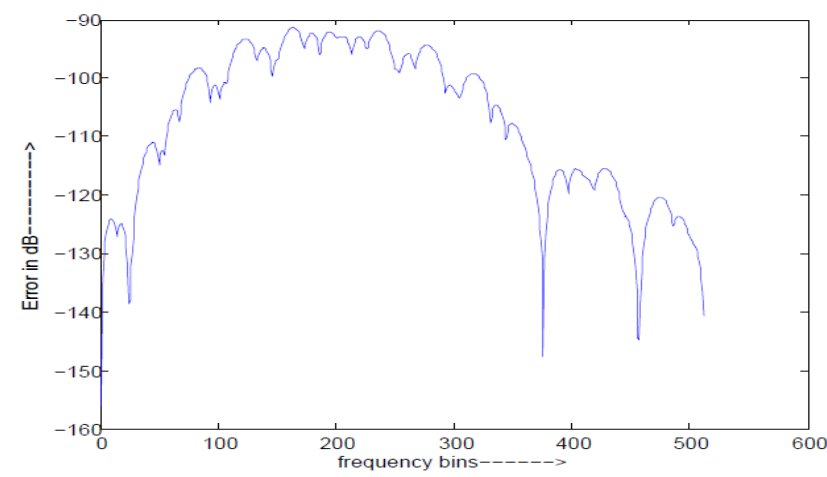

Fig.7 Error plot between Correlation matrix generated for each frequency bin in FPGA Simulation and MATLAB Simulation.

\section{A. Performance}

The proposed architecture could achieve $600 \mathrm{~K}, 8 \times 8$ complex matrix generation per second, which is equivalent to $65.57 \mathrm{fps}$ for a PRF of $4 \mathrm{KHz}, 20 \mathrm{~cm}$ depth, $20 \mathrm{MHz}$ sampling and 61 scan lines per frame. The initial latency is 51.2 usec, as two stage parallel pipeline design is performed (Fig.7). Ping pong buffering approach is followed to address the memory access latency. After initial latency, further there is no latency in generating correlation matrices. The execution time of various processing units are depicted in Fig. 8 and 9. In pipe line-0, FFT and Delay compensator are the arithmetic units and together it takes approximately $24 \mu \mathrm{s}$, which is well within the pipeline- 0 processing time requirements per frequency sub band. In pipe line- 1 the arithmetic units together it takes $0.09 \mu$ s per frequency sub band and total 512 sub bands need to be processed, hence it takes $46.08 \mu \mathrm{s}$ to process all sub bands. Table-3 illustrates the resources utilized and it shows that for the proposed specification, the MVDR Beamformer could be implemented on FPGA.

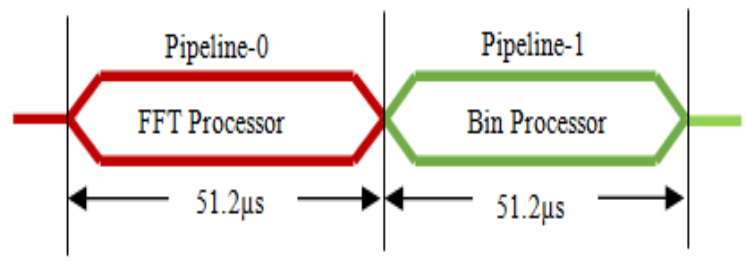

Fig.8 Pipelines in Sub band Processor 


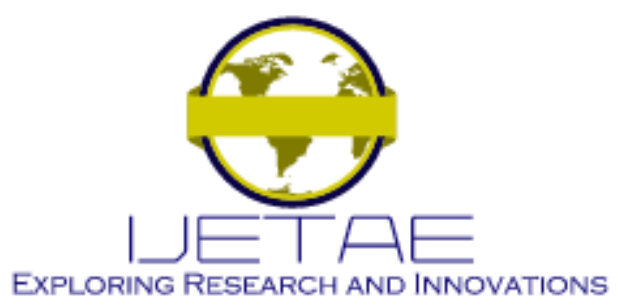

International Journal of Emerging Technology and Advanced Engineering

Website: www.ijetae.com (E-ISSN 2250-2459, Scopus Indexed, ISO 9001:2008 Certified Journal, Volume 11, Issue 06, June 2021)

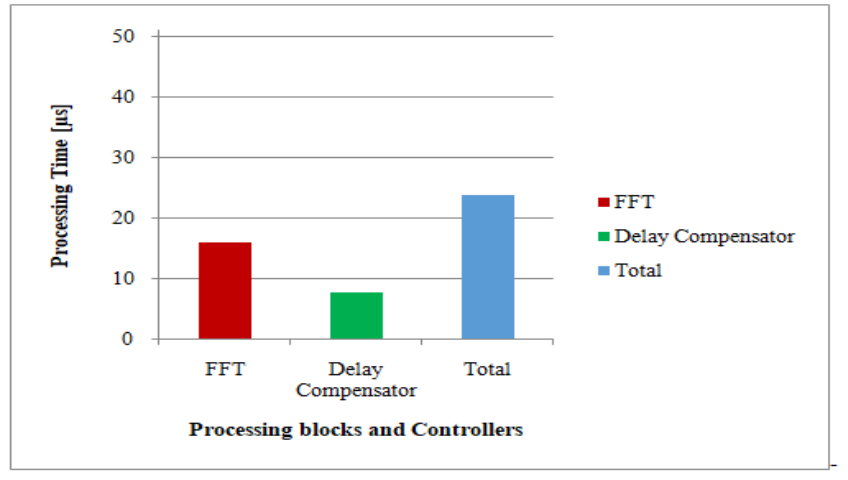

Fig.9 Execution time of various arithmetic units in pipeline-0

of Sub band processor to estimate correlation matrix per frequency sub band.

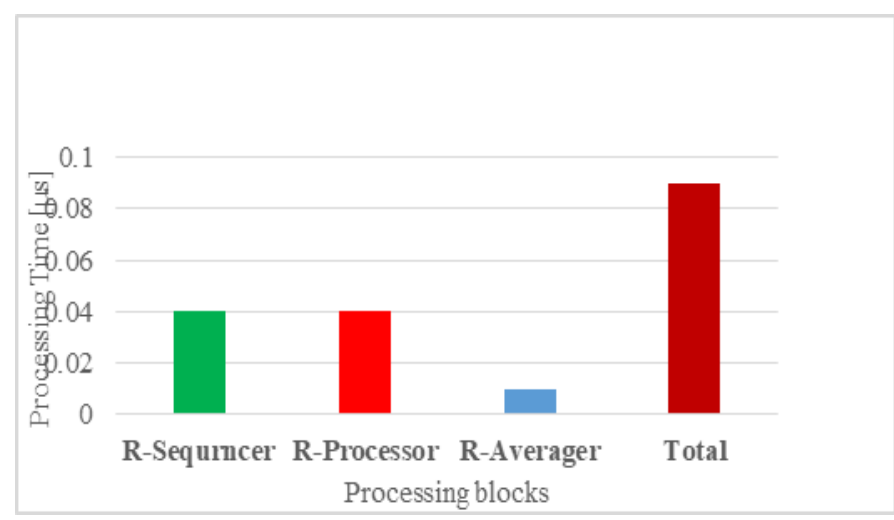

Fig.10 Execution time of arithmetic units per frequency subband in pipe line-1.

\section{B. Computational Parallelism}

In order to satisfy the real-time performance requirement, $321 \mathrm{~K}$ point FFTs, 24 R-Computational processors are operating in parallel. To perform Rcomputation an array of 8 complex multipliers (Fig.6) are utilized to accelerate the performance.

\section{Comparison with Existing Implementations}

In paper [21], a Broadband MVDR Beamformer for medical ultrasound imaging is shown. It demonstrated a simulation model based on Field II, which showed the merits of MVDR for medical ultrasound imaging. But difficulties in practical realization of broad band MVDR in medical ultrasound systems are not discussed. A computationally efficient MVDR beamformer is devised which discussed earlier.
It eliminates the need for computing correlation matrix inverse, but complexity in estimating correlation matrix persists. Article [21] Demonstrated an FPGA based MVDR Beamformer, but complexity in sample correlation matrix calculation is not revealed, rather matrix inversion based on a new technique which is described in an article which is already sited earlier, which is called DCD based method. The computational complexity in estimating correlation matrix ' $\mathrm{R}$ ' is discussed earlier in the article were a GPU based system is utilized and the use of a GPU-optimized MVDR solution for Active SONAR is described. We also discussed an article which demonstrates a parallel implementation of Capon beamformer for Cardiac Imaging on GPU. By utilizing the GPU power they demonstrated real time Cardiac Imaging up to 9 FPS.

In this work we investigated the complexity of computing correlation matrix for each frequency sub bands to perform narrow band beamforming, a parallel pipelined VLSI architecture is designed, implemented on FPGA and performance is investigated. Compared to GPUs most of the Medical Ultrasound Imaging systems are based on FPGAs, due to its high speed I/Os, hardware resources etc. Even though GPUs are having high processing power they lack in IO and memory bandwidth and hence achieving high frame rate is hard. In this work we demonstrated a parallel VLSI architecture for complex correlation matrix generation and its FPGA implementation for achieving high frame rate up to 65 FPS. The memory architectures employed across the sub band processor mitigated the memory access time and hence to achieve the required performance.

In this article we calculated the normalized power of the beam for different angles and compared the same with other architectures. Fig. 11 shows the power of the beam in $\mathrm{dB}$ Vs Angles in Degree for the Sub Array based architecture for the MVDR Beamformer. Fig.12 shows the same for the FPGA Beamformer.

Table-4

Hardware Resources (Virtex6 ML605 Board)

\begin{tabular}{|l|l|l|}
\hline Resources & Utilized & \% Utilized \\
\hline LUT's & 92967 & 23.7 \\
\hline FF's & 97342 & 18 \\
\hline BRAM's & 323.7 & 17.2 \\
\hline
\end{tabular}




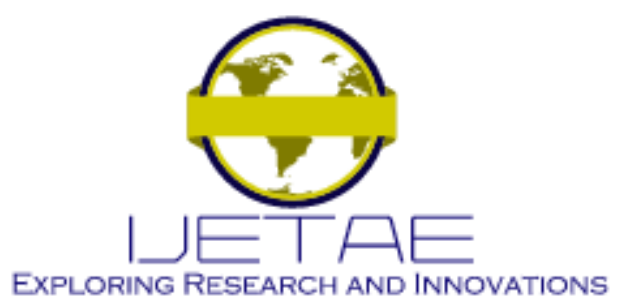

International Journal of Emerging Technology and Advanced Engineering

Website: www.ijetae.com (E-ISSN 2250-2459, Scopus Indexed, ISO 9001:2008 Certified Journal, Volume 11, Issue 06, June 2021)

\section{MATLAB Implementation of the Proposed Architecture}

The MATLAB Implementation of the Sub Array Processor based MVDR Beamformer was completed successfully with the help of the reference function available in the Field II. Simulation of transducer and other required calculations can be done using this particular program which uses the Tupholme-Stepanishen method [21-23]. The Field II is very much powerful enough to calculate the fields for both continuous wave and pulseecho waves. These calculations can be made for large number of transducers. The Field II gives us good images, simulated images with are similar to human tissue images. The various functions used in our architecture implementation is shown in the table below.

Table-5

MATLAB Functions used for Implementation.

\begin{tabular}{|l|l|}
\hline Functions used & $\begin{array}{l}\text { Purpose of the Function in } \\
\text { Implementation. }\end{array}$ \\
\hline hann & $\begin{array}{l}\text { For finding the impulse response of } \\
\text { the transducer. }\end{array}$ \\
\hline xdc_linear_array & $\begin{array}{l}\text { Defining the transducer } \\
\text { parameters. Field II function. }\end{array}$ \\
\hline xdc_impulse & Set the impulse response \\
\hline xdc_excitation & $\begin{array}{l}\text { Set the excitation of the emit } \\
\text { aperture. }\end{array}$ \\
\hline xdc_center_focus & $\begin{array}{l}\text { Set the focus of beam in a } \\
\text { particular direction }\end{array}$ \\
\hline xdc_focus & $\begin{array}{l}\text { Set the focus of beam in a } \\
\text { particular direction }\end{array}$ \\
\hline calc_scat & Calculate the received response \\
\hline xdc_focus_times & Array Signal Generation \\
\hline calc_scat_multi & Calculations \\
\hline
\end{tabular}

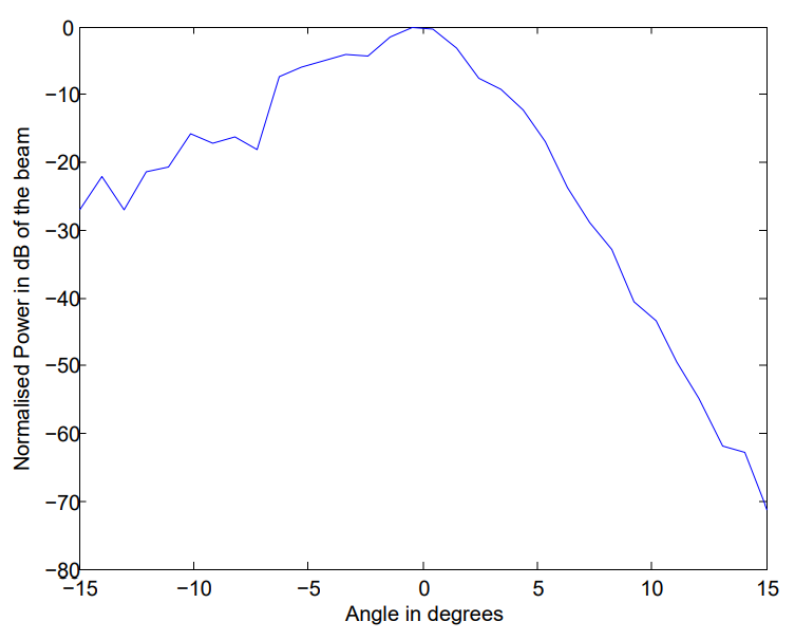

Fig.11 Sub Array based MVDR Beamformer Normalized Power.

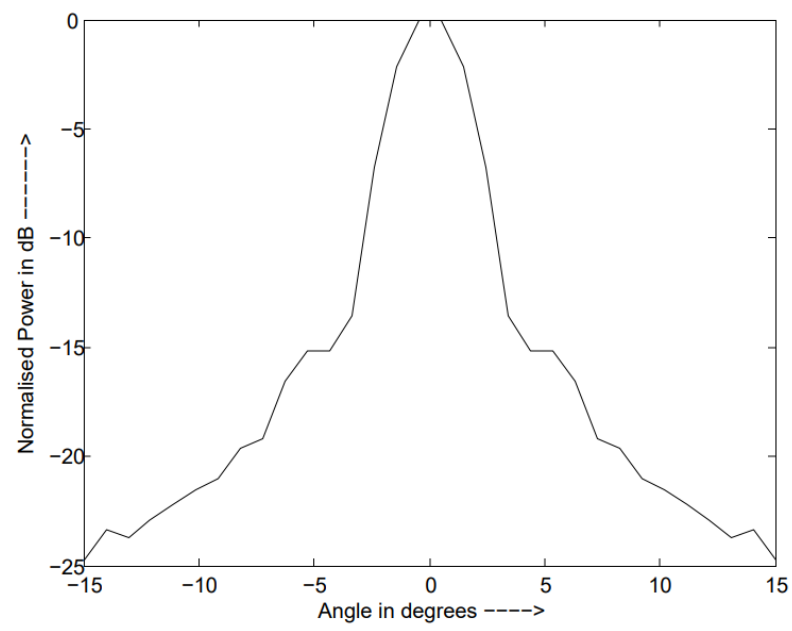

Fig.12 FPGA Implementation of MVDR Beamformer Normalized Power.

\section{CONCLUSION}

In this paper a parallel architecture for sub band processor in frequency domain MVDR Beamformer have been presented and the architecture has successfully been implemented on FPGA. 


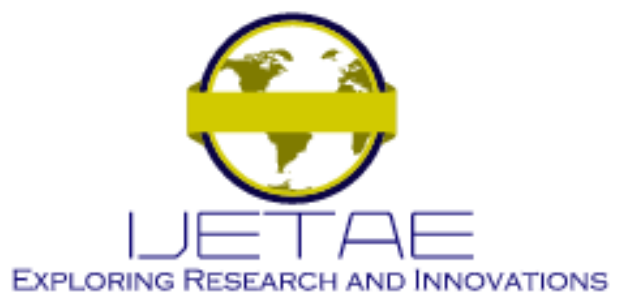

International Journal of Emerging Technology and Advanced Engineering

Website: www.ijetae.com (E-ISSN 2250-2459, Scopus Indexed, ISO 9001:2008 Certified Journal, Volume 11, Issue 06, June 2021)

The complexity of estimating correlation matrix is revealed and the architecture could attain a real-time performance of $65.57 \mathrm{fps}$ to image up to $20 \mathrm{~cm}$ depth, with a PRF of $4 \mathrm{KHz}$. The design was successfully ported on Xilinx Virtex-6, ML605 EVM and results were validated with MATLAB Field II counterpart reference model. The architecture is running in various clock domains and the resource utilizations can be compromised as system implementation is concerned.

\section{LIMITATIONS AND FUTURE SCOPE}

The major limitation of the architecture proposed is the computational complexity associated with it. The FPGA implementation of the architecture becomes almost impossible with the complexity which increases latency and which in turn add to the lower frame rates and decreased resolution of the images. We have shown the implementation for 32 channels with sub array length 8, and the real time computational cost is handled by making use of flexibility in building a parallel architecture on FPGA.

Solution of reducing this computation complexity of the MVDR Beamformer is to remove the need of calculations which involves the averaging, matrix multiplication and computation of the co-variance for the calculation of the adaptive weights. A parallel beamformer architecture for adaptive beamformer will be a solution for this problem which does the calculation by a single transmit and while on reception beamforming is done on all possible theta directions in a parallel manner.

\section{Acknowledgement}

As per order No 9(1)/2014 -MDD dated 15-12-2014, this study is supported by the Microelectronics Division of the Ministry of Electronics and Information Technology, Government of India, under the SMDP-C2SD Project.

\section{REFERENCES}

[1] Hema, N., Kidav, J., \& Lakshmi, B. (2015). VLSI Architecture for Broadband MVDR Beamformer. Indian Journal Of Science And Technology.

[2] Jongin Park, Seok-Min Wi, and Jin S. Lee," Computationally Efficient Adaptive Beamformer for Ultrasound Imaging Based on QR Decomposition",IEEE TRANSACTIONS ON ULTRASONICS, FERROELECTRICS, AND FREQUENCY CONTROL, VOL. 63, NO. 2, FEBRUARY 2016.

[3] O. L. Frost III(1972), "An algorithm for linearly constrained adaptive array processing" Proc. IEEE, vol. 60, no. 8, pp. 926-935
[4] Jo Inge Buskenes, Jon Petter Åsen, Carl-Inge Colombo Nilsen and Andreas Austeng,"An Optimized GPU Implementation of the MVDR Beamformer for Active Sonar Imaging",IEEE JOURNAL OF OCEANIC ENGINEERING, VOL. 40, NO. 2, APRIL 2015

[5] Jon Petter Åsen, Jo Inge Buskenes, Carl-Inge Colombo Nilsen, Andreas Austeng, and Sverre Holm, " Implementing Capon Beamforming on a GPU for Real-Time Cardiac Ultrasound Imaging", IEEE Transactions on Ultrasonics, Ferroelectrics, and Frequency Control, vol. 61, no. 1, January 2014 0885-3010 @ 2014 IEEE.

[6] A. Mann and W. F. Walker(1988), "A constrained adaptive beamformer for medical ultrasound: Initial results", in Proc. IEEE Ultrason. Symp., Oct. 2002, pp. 1807-1810.

[7] T J Shan and T. Kailath(1985), "Adaptive Beamforming for Coherent Signals and Interference",IEEE Trans.Acous., Speech, Sig Pro., vol.33. no .3, pp, 527-536.

[8] M. Sasso and C. Cohen-Bacrie(1980-1983), "Medical ultrasound imaging using the fully adaptive beamformer" in Proc. Int. Conf. Acoust. Speech Signal Process. (ICASSP), Mar. 2005, pp. 489-492.

[9] Kidav, N. M Sivamangai, M. P Pillai, Subash Raja M. "Architecture and FPGA Prototype of CYCLE STEALING DMA ARRAY SIGNAL PROCESSOR FOR ULTRASOUND SECTOR IMAGING SYSTEMS" , Microprocessors and Microsystems, 2018

[10] Chris Dick, Fred Harris, M J. Capon(1969),“ High-resolution frequency-wavenumberspectrum analysis," Proc. IEEE, vol. 57, no. 8, pp.1408-1418.

[11] "Field II Ultrasound Simulation Program", Field-ii.dk, 2016. [Online]. Available: http://field-ii.dk/.

[12] S. G. Sreejeesh, R. Sakthivel and J. U. Kidav, "Superior Implementation of Accelerated QR Decomposition for Ultrasound Imaging," in IEEE Access, vol. 8, pp. 156244-156260, 2020, doi: 10.1109/ACCESS.2020.3017558

[13] Iroslav Pajic and Dragan Vuletic, Implementing a realtime beamformer on an FPGA Platform. Xcell Journal, First Quarter 2007.

[14] Robert Hamill, John V. McCanny, and Richard L. Walke, Online CORDIC Algorithm and VLSI Architecture for Implementing QR Array processors.IEEE TRANSACTIONS ON SIGNAL PROCESSING, VOL. 48, NO. 2, FEBRUARY 2000.

[15] GJohan-Fredrik Synnevåg, Andreas Austeng, and Sverre Holm, " Benefits of Minimum-Variance Beamforming in Medical Ultrasound Imaging", IEEE Transactions on Ultrasonics, Ferroelectrics, and Frequency Control, vol. 56, no. 9, September 2009.

[16] I. K. Holfort, F. Gran and J. A. Jensen, "P2B-12 Minimum Variance Beamforming for High Frame-Rate Ultrasound Imaging," 2007 IEEE Ultrasonics Symposium Proceedings, 2007, pp. 1541-1544, doi: 10.1109/ULTSYM.2007.388.

[17] Iben Kraglund Holfort, Fredrik Gran and Jørgen Arendt Jensen "Broadband Minimum variance beamforming for ultrasound Imaging"IEEE Transactions on Ultrasonics, Ferroelectrics, and Frequency Control, vol. 56,No 2, February 2009

[18] S. Wang, Y. Tien, Y. Hwang, J. Lin and G. Wu, "MVDR based adaptive beamformer design and its FPGA implementation for ultrasonic imaging," 2016 IEEE Asia Pacific Conference on Circuits and Systems (APCCAS), 2016, pp. 143-145, doi: 10.1109/APCCAS.2016.7803917. 


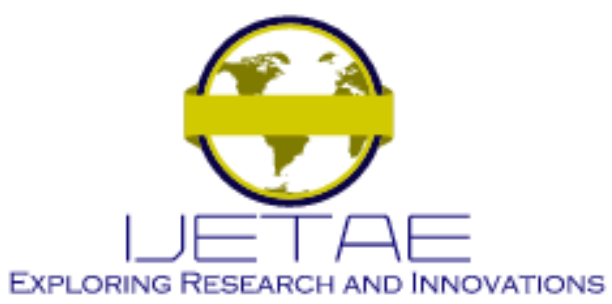

International Journal of Emerging Technology and Advanced Engineering Website: www.ijetae.com (E-ISSN 2250-2459, Scopus Indexed, ISO 9001:2008 Certified Journal, Volume 11, Issue 06, June 2021)

[19] Jie Liu, Ben Weaver, Yuriy Zakharov, George White, An FPGA based MVDR beamformer using dihotomous coordinate descent iterations.ICC 2007 proceedings.

[20] Jayaraj U. Kidav, N.M. Siva Mangai, M.P. Pillai. "Design of AWC core using DCD iterations for MVDR beamformer" , Microprocessors and Microsystems, 2020
[21] G.E. Tupholme: Generation of acoustic pulses by baffled plane pistons, Mathematika 16, pp. 209-224, 1969.

[22] P.R. Stepanishen: The time-dependent force and radiation impedance on a piston in a rigid infinite planar baffle, J.Acoust.Soc.Am. 49 (3), pp. 841-849, 1971A.

[23] P.R. Stepanishen: Transient radiation from pistons in a infinite planar baffle, J.Acoust.Soc.Am. 49, pp. 1627-1638, 1971B. 